\title{
Radiation sparing of cerebral cortex in brain tumor patients using quantitative neuroimaging
}

Roshan A. Karunamuni ${ }^{1}$, PhD; Kevin L. Moore ${ }^{1}$, PhD; Tyler M. Seibert ${ }^{1}, \mathrm{MD}, \mathrm{PhD}$; Nan $\mathrm{Li}^{1}, \mathrm{PhD}$; Nate S. White ${ }^{2}, \mathrm{PhD}$; Hauke Bartsch ${ }^{2}, \mathrm{PhD}$; Ruben Carmona ${ }^{1}, \mathrm{MD}$ MAS; Deborah Marshall ${ }^{1}, \mathrm{BA} ;$ Carrie R. McDonald ${ }^{3}$, PhD; Nikdokht Farid ${ }^{2}, \mathrm{MD}$; Anithapriya Krishnan ${ }^{2}, \mathrm{PhD}$, Joshua Kuperman ${ }^{2}, \mathrm{PhD}$; Loren K. Mell ${ }^{1}, \mathrm{MD}$; James Brewer ${ }^{2}$, MD PhD; Anders M. Dale ${ }^{2}, \mathrm{PhD}$; Vitali Moiseenko ${ }^{1}, \mathrm{PhD}$; Jona A. Hattangadi-Gluth ${ }^{1}$, MD

${ }^{1}$ Department of Radiation Medicine and Applied Sciences, University of California San Diego, La Jolla, California

${ }^{2}$ Department of Radiology, University of California San Diego, La Jolla, California ${ }^{3}$ Department of Psychiatry, University of California San Diego, La Jolla, California

Running title: Cortical radiation sparing in glioma Keywords: cortex, glioma, radiation sparing, OAR Manuscript word count: 2,981

Total figures and table count: 5 


\section{Corresponding Author:}

Jona A. Hattangadi-Gluth, MD

Department of Radiation Medicine and Applied Sciences 3855 Health Sciences Drive

La Jolla, CA 92093

jhattangadi@ucsd.edu / (858)-822-6040 / (858) 246-1505 (fax) 


\section{Abstract}

Background and Purpose: Neurocognitive decline in brain tumor patients treated with radiotherapy (RT) may be linked to cortical atrophy. We developed models to determine radiation treatment-planning objectives for cortex, which were tested on a sample population to identify the dosimetric cost of cortical sparing.

Material and Methods: The relationship between the probability of cortical atrophy in fifteen high-grade glioma patients at 1-year post-RT and radiation dose was fit using logistic mixed effects modeling. Cortical sparing was implemented using two strategies: region-specific sparing using model parameters, and non-specific sparing of all normal brain tissue.

Results: A dose threshold of 28.6 Gy was found to result in a $20 \%$ probability of severe atrophy. Average cortical sparing at 30 Gy was greater for region-specific dose avoidance (4.6\%) compared to non-specific (3.6\%). Cortical sparing resulted in an increase in heterogeneity index of the planning target volume (PTV) with an average increase of $1.9 \%$ (region-specific) and $0.9 \%$ (nonspecific).

Conclusions: We found RT doses above 28.6 Gy resulted in a greater than $20 \%$ probability of cortical atrophy. Cortical sparing can be achieved using regionspecific or non-specific dose avoidance strategies at the cost of an increase in the dose heterogeneity of the PTV. 


\section{Introduction}

Long-term neurocognitive dysfunction is an unfortunate consequence of brain radiotherapy $(\mathrm{RT})$ [1-3]. The quantitative analysis of normal tissue effects in the clinical (QUANTEC) review of RT-induced brain injury supports the need for conclusive evidence relating radiotherapy and neurocognitive decline, citing imaging changes as a potential early identifier of clinically relevant endpoints [4]. Imaging provides robust estimates of treatment-induced toxicity before detection by routine clinical methods [5]. Continued study into the effects of RT on normal brain can provide dosimetric objectives for organs-at-risk (OAR) that were not previously considered to be important.

We recently published our findings on dose-dependent cortical thinning in high-grade glioma (HGG) patients one year after fractionated partial-brain RT [6]. The magnitude of atrophy parallels one-year atrophy rates in neurodegenerative diseases, with greater effects seen in regions of the cortex treated with higher doses. In addition, recent studies suggest that radiation results in decreased density of vascular endothelial cells [7] and an increased rate of tau protein misfolding within cultured primary neurons [8]. Vascular degeneration and abnormal protein accumulation have been reported in several forms of clinical dementia $[9,10]$, suggesting that the neurocognitive sequelae observed in RT patients may be linked to radiation effects in cortical tissue.

Dose guidelines and planning constraints for OAR in the brain are widely available in the literature [11-13], and build on work by Emami et al. [14]. These parameters serve as guidelines for physicians to predict the relative safety of 
proposed treatment plans. However, the division of the brain into OAR remains crude with no region-specific objectives for subcortical white matter, cortical gray matter, and deep lying gray matter - all of which may have varying sensitivity to radiation dose. The brain is a highly complex organ, and tissue that is not currently considered critical may be involved in the regulation of several cognitive processes [15]. Compared with neurosurgery [16,17], radiation-planning pays little attention to the functional importance of different areas of the brain.

In this study, we explore the feasibility of employing RT dose avoidance of cortex in patients treated for HGG using inverse-optimized planning. Dose objectives were formulated using complication probability modeling of the effect of radiation dose on changes in cortical thickness. Cortical thinning, detected using volumetric magnetic resonance imaging (MRI), was selected as an imaging biomarker of cortical damage due to its extensive application in the neuroimaging literature [18-20], including studies on Alzheimer's disease [21] and vascular dementia[22]. Cortical dose avoidance strategies were tested on the sample population to identify any dosimetric cost to imposing cortical dose constraints.

\section{Materials and Methods}

Patients

The cohort consists of fifteen consecutively treated HGG patients who underwent fractionated partial brain RT at the University of California San Diego between 2011- 2013 [6]. To meet inclusion criteria, patients must have undergone high-resolution brain MRI prior to RT and at 1-year post-RT. Among 
72 HGG patients treated with partial brain RT from 2011- 2013, 22 patients had the necessary imaging scan dates. Of these 22 patients, seven were removed due to image misregistration and severe mass-effect. The remaining 15 cases were used for this analysis. This study was approved by the institutional review board.

\section{MR Imaging}

MR imaging was performed on a 3T Signa Excite HDx scanner (GE Healthcare, Milwaukee, Wisconsin) equipped with an 8-channel head coil. The imaging protocol included pre- and post-contrast 3D volumetric T1-weighted inversion recovery spoiled gradient-echo sequence (TE, $2.8 \mathrm{~ms}$; TR, $6.5 \mathrm{~ms}$; TI, $450 \mathrm{~ms}$ ) and a 3D T2-weighted FLAIR sequence (TE, $126 \mathrm{~ms}$; TR, $6000 \mathrm{~ms}$; TI, $1863 \mathrm{~ms})$. All MR images were corrected for geometric distortions due to susceptibility, gradient nonlinearities, and eddy currents [23]. Patient motion between sequences was corrected using rigid-body registration algorithms developed in MATLAB (Mathworks, Natick, Massachusetts). The treatment planning CT images were rigidly co-registered to the pre-RT T1-weighted precontrast images [24] and the transformation matrix was used to project the radiation dose maps, to the MR images [6].

\section{Cortical Thinning}

Cortical thickness was measured using Freesurfer (version 5.3; available at http://surfer.ndm.harvard.edu), as previously described [6]. Vertices 
corresponding to the cortical surface were grouped into 1 Gy dose bins. Surgical scar, tumor, tumor beds and resection cavities were manually censored from analyses. The 1-year percent cortical thinning for each dose bin was calculated as the vertex-averaged ratio of change in thickness (pre RT - post-RT) to the baseline value (pre-RT).

\section{NTCP modeling of cortical atrophy}

Three grades of complication were used to classify the 1-year percent cortical thinning in this study: $>2 \%$ (grade 1$),>3 \%$ (grade 2 ), and $>5 \%$ (grade 3). These cutoffs were chosen to reflect the average percent thinning reported in the literature for mild cognitive impairment (MCl), Alzheimer's disease (AD), and severe Alzheimer's disease, respectively [25]. The effect of dose on the incidence of each grade of complication in the study sample was fit using a logistic mixed effects model. A patient-specific intercept and slope were tested to control for correlated data points within patients. Main effects were significant at $p<0.05$. Estimates of main fixed effects were used to calculate $R_{X}$, the radiation dose corresponding to a $\mathrm{X} \%$ incidence of complication, and $\mathrm{y}$, the normalized slope of the dose-response curve, using:

$$
\begin{gathered}
R_{X}=\left[-\ln \left(\frac{1}{\left(\frac{X}{100}\right)}-1\right)-\beta_{0}\right] / \beta_{\beta_{1}} \\
\gamma=-\beta_{0} / 4,(1)
\end{gathered}
$$


$\beta_{0}$ is the log odds at a dose of $0 \mathrm{~Gy}, \beta_{1}$ is the change in the log odds per unit increase in dose. We used bootstrap resampling with 1000 samples and replacement to obtain $95 \%$ confidence intervals for the model parameters. Statistical analyses were conducted in R ("Ime4" package, version 1.1-7).

Importing cortical segmentation into treatment planning software

Radiation structure files containing the original structure set and cortical segmentations from Freesurfer were generated. Radiation treatment files were imported into a research Eclipse ${ }^{\mathrm{TM}}$ treatment planning system (Varian Medical Systems, Palo Alto, CA) for re-planning. Contours were visually inspected in the treatment planning software to ensure that the cortex was properly segmented. Subcortical white matter and hippocampus segmentation volumes were also imported from Freesurfer into the treatment planning system. Cortical, hippocampal, and white matter segmentations were processed to remove regions that overlapped with the planning target volume (PTV). An example of the updated structure set is shown in Supplementary Figure 1. Doses were calculated on a $2.5 \times 2.5 \times 1.25 \mathrm{~mm}$ grid using the analytic anisotropic algorithm (AAA) algorithm that includes tissue heterogeneity corrections.

Re-planning using cortical NTCP constraint

Patients were planned with and without cortical NTCP dose constraints to determine whether cortical radiation sparing is possible. Dose constraints to the target, planning treatment volume (PTV), and standardized OAR, such as brain 
stem and optic nerve, matched those used clinically and conform to the guidelines set by RTOG 0825 [26]. The primary objective was to maintain at least $95 \%$ volume coverage to the PTV at $100 \%$ prescription dose. All plans were matched on this objective. Cortical dose sparing was achieved by minimizing cortical volume above $\mathrm{R}_{20}$, dose corresponding to $20 \%$ probability of complication, for grade 3 thinning. $R_{20}$ for grade 3 was chosen as it represented a tradeoff between imposing too stringent an optimization requirement (such as $R_{50}$ for grade 3$)$, versus a lenient approach $\left(R_{50}\right.$ for grade 1$)$, which would allow for too high a probability of cortical thinning. Cortical sparing was quantified by calculating $V_{Y}$, defined as the volumetric percentage receiving doses greater than $Y$ Gy. Dosimetric indices such as mean dose, $D_{Z}$ (defined as the minimum dose to the hottest $Z \%$ of the volume), heterogeneity index $\left(H I\right.$, defined as $\left(D_{2}-\right.$ $D_{98} / 60$ Gy ${ }^{*} 100 \%$ ) were used to quantify the effect of imposing cortical dose constraints on the PTV. An alternate dose avoidance strategy of minimizing $R_{20}$ of all normal brain was tested to determine whether cortical sparing could be achieved by non-specific avoidance of normal brain tissue.

\section{Results}

The cohort consisted of 10 males and 5 females with a median age of 60 years (range 40 - 77). Most patients had glioblastoma with a median preoperative tumor size and planning target volume of $3.3 \mathrm{~cm}$ (range: $1.0-7.7 \mathrm{~cm}$ ) and $162.7 \mathrm{cc}$ (range: $59.8-571.3 \mathrm{cc}$ ), respectively. Patients were treated using a 6 MV TrueBeam (Varian Medical Systems, Palo Alto, CA) linear accelerator with 
a 120-leaf Millennium multileaf collimator to a prescription dose of 59.4-60 Gy in 33 or 30 fractions, respectively. 13 patients were treated with 6 to 8 non-coplanar field, fixed-gantry, sliding-window IMRT, and 2 patients were treated with two coplanar arc VMAT. These planning parameters were not altered for the replanning study. The most common (33\%) chemotherapy treatment was concurrent and adjuvant Temozolomide. Five patients had intervening surgeries between the two time points, four of which were due to tumor recurrence.

The predicted relationship between radiation dose and probability of complication was derived using estimates from the logistic mixed effects model, shown in Figure 1. The curves in red, green, and blue represent the probability of developing cortical atrophy at grades 1,2, and 3 respectively. The shaded regions represent the upper and lower bounds of the $95 \%$ confidence interval. The probability of atrophy, irrespective of grade, is significantly associated with radiation dose $(p<0.001)$.

Estimates for $\gamma, \mathbf{R}_{50}, \mathbf{R}_{20}$, and $\mathbf{R}_{10}$ were derived using logistic mixed effect model parameters and tabulated in Table 1. The values of $\gamma$, which represent the normalized slope of the probability function at a $50 \%$ complication probability, were found to be $0.29,0.62$ and 1.24 for grades 1,2 and 3 respectively. For every $1 \%$ increase in dose, the increase in complication probability is greatest for grade 3 . The values of $R_{50}, R_{20}$, and $R_{10}$ increased with increasing severity of complication grade. $R_{20}$ and $R_{10}$ were not calculated for grade 1 , as the probability at baseline is higher than $20 \%$. $R_{20}$ for grade 3 was calculated to be 28.6 Gy. 
An example of cortical radiation sparing using a region-specific dose avoidance strategy in a single subject is shown in Figure 2. Axial slices of the planning CT volume overlaid with cortical contours and radiation dose color maps, ranging from 30 to $65 \mathrm{~Gy}$, are shown with and without cortical sparing. The dose fall-off from the $60+$ Gy plateau is sharper after implementation of cortical dose constraints. Regions of cortex distal to the PTV that were receiving higher than 30 Gy are lowered under that threshold. PTV coverage was maintained, at the cost of an increase in $\mathrm{HI}$ from 8.21 to $10.44 \%$. The change in mean dose to the standardized OAR was minimal. However, cortical sparing resulted in the incidental change in dose distribution of the cerebral white matter and hippocampus with dose-averaged changes in $V_{Y}$ of -1.2 and $-0.8 \%$, respectively.

Table 2 summarizes the changes in dosimetric indices for the entire patient cohort using both a region-specific dose avoidance strategy for the cortex and a non-specific dose avoidance strategy for all normal brain tissue. Cortical $V_{Y}$ decreased, or remained unchanged, for all dose levels between 10 and 50 Gy irrespective of dose avoidance strategy. Average cortical sparing at 30 Gy was greater for region-specific dose avoidance (4.6\%) compared to non-specific (3.6\%). Negligible changes were observed in $\mathrm{V}_{60}$ for all OAR. The magnitude of decrease in $\mathrm{V}_{\mathrm{y}}$ was greater using the region-specific strategy for doses greater than $30 \mathrm{~Gy}$, whereas the converse is true for doses less than $30 \mathrm{~Gy}$. This is illustrated in Figure 3, which shows a histogram of the per-voxel probability of grade 3 atrophy using the original and re-plans for a single patient. The probability is calculated for each voxel using the parameters from the previously 
described logistic mixed effects model. The probability-weighted number of voxels at risk of grade 3 atrophy (defined as the product of probability and number of voxels, summed over all probability bins) was calculated for the original, non-specific, and region-specific plans as $68.2,60.6$, and 53.5 thousand voxels respectively, corresponding to $71.6,63.9$ and $56.4 \mathrm{~cm}^{3}$ respectively.

The implementation of both dose avoidance strategies resulted in a decrease in $V_{Y}$ of the white matter and hippocampus. However, the magnitude of sparing of these OAR was larger when using a non-specific approach. As in the previously described example, imposing cortical dose constraints resulted in a change in the dose distribution to the PTV. The change in PTV dose distribution was, however, smaller in magnitude using the non-specific approach.

\section{Discussion}

In this work, we demonstrate that the relationship between incidence of cortical atrophy and radiation dose can be modeled using a logistic function, irrespective of the grading threshold used to binarize the magnitude of cortical thinning. Our results showed that doses above 28.6 Gy resulted in a $20 \%$ or greater probability of severe (grade 3 ) cortical thinning. Radiation sparing of cortex above this dose can be achieved using several dose avoidance strategies at the cost of a modest increase in the dose heterogeneity of the PTV.

The relationship between probability of cortical atrophy and radiation dose is described in Figure 1 and Table 1. The relationship was modeled using a logistic function for all three grades of complication. The model fit using grade 1 
$(2 \%)$ atrophy suggests that even low radiation doses (less than $1 \mathrm{~Gy}$ ) result in a complication probability of $22 \%$. This offset probability of cortical atrophy may be indicative of a systemic decrease in cortical thickness, mediated by chemotherapy, surgery and inflammation. Offset values have also been observed in other normal tissue complication probability models [27], and have been attributed to the effects of underlying disease and treatment other than radiation.

Two strategies were investigated for implementing cortical dose avoidance. A region-specific strategy, which explicitly attempted to minimize cortical dose exposure, was better able to spare cortex at doses greater than 30 Gy than a more general approach of minimizing dose to the entire brain (Table 2 and Figure 2). However, the non-specific approach did improve sparing of white matter and hippocampus, both of which have been implicated as important OAR $[28,29]$.

The imposition of a non-specific dose avoidance strategy may represent a more conservative approach to cortical dose sparing. While this approach cannot replicate the high-dose cortical sparing that a targeted strategy would yield, it may represent a compromise that effectively spares all brain tissue types. There is little data to support whether sparing of particular critical brain structures takes precedence over others. In addition, a non-specific approach is more easily implemented, as it uses structures that are already part of the clinical workflow. It should be highlighted, however, that region-specific avoidance strategy offers the same percentage-wise improvements ( $13 \%$ reduction in volume of tissue to 
experience severe atrophy) as non-specific dose avoidance offered over the original clinical planning sample.

The consequence of cortical radiation sparing is a redistribution of the dose to the PTV. In particular, the cortical dose constraints result in a modest decrease in $D_{98}$ and an increase in $D_{2}$ of the PTV dose distribution. If the regions getting marginally hotter are within the gross tumor volume (GTV), this seems acceptable to prevent the unnecessary radiation of cortex. For example, a clinical trial is currently underway to determine if dose escalated RT improves overall survival and is testing a prescribed dose of 75 Gy to the GTV (NRG-BN001 [30]). By comparison, the maximum $D_{2}$ using either dose avoidance strategy in our study was 66.7 Gy (111\%).

Alternatively, if the maximum point dose lies outside the GTV, the unnecessary radiation of regions within the PTV that may consist of normal tissue will have to be weighed against a potential reduction in high-dose irradiation of the cortex. This decision will most likely be influenced by several factors, such as the anatomic location of tumor and maximal doses. The QUANTEC radiation guidelines for normal tissue sparing state that doses between 60 and 72 Gy, resulted in a probability of radiation necrosis between 3 and $5 \%$. Maximum point doses less than 72 Gy may, therefore, represent an acceptable amount of radiation damage for potential sparing of neurocognitive function through cortical dose avoidance.

This study has limitations. The patient sample is small; however we are well-powered to perform this analysis as each patient provided multiple data 
points (60 dose bins). The repeated measures were consequently accounted for using mixed effects modeling. The effect of intervening surgeries or tumor recurrence also remains unclear. However, all surgical cavities were censored and the prevalence of cortical atrophy in areas of normal-appearing brain suggests that these effects are secondary to that of the radiation dose. In addition, dose thresholds for cortical sparing were based on cortical atrophy, and not neurocognitive function. Cortical thinning has been observed and reliably measured in several neurodegenerative diseases. The relationship between cortical thinning post-RT and cognitive decline is currently being studied prospectively at our institution. As previously described, cortical thinning of this magnitude is beyond normal age-related atrophy and may therefore be associated with an underlying pathological process.

Finally, the inverse planning process can be dependent on how the priorities for various objectives are balanced, which may, in turn, be dependent on the particular planner. However, the plans used as the reference (original treatment plan) in our study were clinical plans approved for treatment by the physician and so can be assumed to be the best available.

Radiation dose to the uninvolved cerebral cortex can be reduced in patients undergoing fractionated brain RT while maintaining clinically required PTV coverage. A dose cut-off value of 30 Gy was identified above which radiation doses will result in a $20 \%$ or greater probability of observing cortical atrophy similar in magnitude to the regional atrophy observed in severe neurodegenerative diseases. Cortical volume percentage above 30 Gy was 
successfully reduced at the cost of an increase in the maximum dose and heterogeneity of dose distribution of the PTV. Future studies will attempt to determine whether cortical radiation sparing results in a demonstrable improvement in the neurocognitive function and quality of life of RT patients.

\section{Acknowledgements}

This work was partially supported by the following grants: National Institutes of Health Grant KL2RR031978, KL2TR00099, UL1TR000100, American Cancer Society Pilot Award ACS-IRG \#70-002, National Science Foundation Grant 1430082. 


\section{References}

[1] Copeland DR, DeMoor C, Moore BD, Ater JL. Neurocognitive development of children after a cerebellar tumour in infancy: A longitudinal study. J Clin Oncol. 1999;17(11):3476-3486.

[2] Meyers CA, Brown PD. Role and relevance of neurocognitive assessment in clinical trials of patients with CNS tumors. J Clin Oncol. 2006;24(8):13051309. doi:10.1200/JCO.2005.04.6086.

[3] Greene-Schloesser DM, Moore E, Robbins ME. Molecular pathways: Radiation-induced cognitive impairment. Clin Cancer Res. 2013;19(9):2294-2300. doi:10.1158/1078-0432.CCR-11-2903 .

[4] Lawrence YR, Li XA, el Naqa I, et al. Radiation Dose-Volume Effects in the Brain. Int J Radiat Oncol. 2010;76(3):S20-S27. doi:10.1016/j.jrobp.2009.02.091.

[5] Benczik J, Tenhunen M, Snellman M, et al. Late radiation effects in the dog brain: correlation of MRI and histological changes. Radiother Oncol. 2002;63(1):107-120. doi:10.1016/S0167-8140(02)00028-2.

[6] Karunamuni R, Bartsch H, White NS, et al. Dose-dependent Cortical Thinning After Partial Brain Irradiation in High-grade Glioma. Int $J$ Radiat Oncol Biol Phys. 2015;In Press. doi:10.1016/j.jirobp.2015.10.026.

[7] Lyubimova N, Hopewell JW. Experimental evidence to support the hypothesis that damage to vascular endothelium plays the primary role in the development of late radiation-induced CNS injury. Br J Radiol. 2004;77(December 2003):488-492. doi:10.1259/bjr/15169876.

[8] Li L, Wang W, Welford S, Zhang T, Wang X, Zhu X. lonizing radiation causes increased tau phosphorylation in primary neurons. J Neurochem. 2014;131:86-93. doi:10.1111/jnc.12769.

[9] Buee L, Hof $P$, Delacourte A. Brain Microvascular Changes in Alzheimer's Disease and Other Dementias. Ann N Y Acad Sci. 1997;826:7-24. http://onlinelibrary.wiley.com/doi/10.1111/j.1749-6632.1997.tb48457.x/full. 
[10] Spillantini MG, Goedert M. Tau protein pathology in neurodegenerative diseases. Trends Neurosci. 1998;21(10):428-433. doi:10.1016/S01662236(98)01337-X.

[11] Marks LB, Yorke ED, Jackson A, et al. Use of Normal Tissue Complication Probability Models in the Clinic. Int J Radiat Oncol Biol Phys. 2010;76(3 SUPPL.). doi:10.1016/j.jrobp.2009.07.1754.

[12] Shaffer R, Nichol AM, Vollans E, et al. A Comparison of Volumetric Modulated Arc Therapy and Conventional Intensity-Modulated Radiotherapy for Frontal and Temporal High-Grade Gliomas. Int J Radiat Oncol Biol Phys. 2010;76(4):1177-1184. doi:10.1016/j.jirobp.2009.03.013.

[13] Scoccianti S, Detti B, Gadda D, et al. Organs at risk in the brain and their dose-constraints in adults and in children : A radiation oncologist ' $s$ guide for delineation in everyday practice. Radiother Oncol. 2015;114(2):230238. doi:10.1016/j.radonc.2015.01.016.

[14] Emami B, Lyman J, Brown a, et al. Tolerance of normal tissue to therapeutic irradiation. Int J Radiat Oncol Biol Phys. 1991;21(1):109-122. doi:10.1016/0360-3016(91)90171-Y.

[15] Monsell S. Task switching. Trends Cogn Sci. 2003;7(3):134-140. doi:10.1016/S1364-6613(03)00028-7.

[16] Sincoff EH, Tan Y, Abdulrauf SI. White matter fiber dissection of the optic radiations of the temporal lobe and implications for surgical approaches to the temporal horn. J Neurosurg. 2004;101(5):739-746. doi:10.3171/jns.2004.101.5.0739.

[17] Pantelis E, Papadakis N, Verigos K, et al. Integration of functional MRI and white matter tractography in stereotactic radiosurgery clinical practice. Int $\mathrm{J}$ Radiat Oncol Biol Phys. 2010;78(1):257-267. doi:10.1016/j.jijrobp.2009.10.064.

[18] Makris N, Biederman J, Valera EM, et al. Cortical Thinning of the Attention and Executive Function Networks in Adults with AttentionDeficit/Hyperactivity Disorder. Cereb Cortex. 2007;17(6):1364-1375. doi:10.1093/cercor/bhl047. 
[19] Chang Y-L, Jacobson MW, Fennema-Notestine C, et al. Level of Executive Function Influences Verbal Memory in Amnestic Mild Cognitive Impairment and Predicts Prefrontal and Posterior Cingulate Thickness. Cereb Cortex. 2010;20(6):1305-1313. doi:10.1093/cercor/bhp192.

[20] McCauley SR, Wilde EA, Merkley TL, et al. Patterns of Cortical Thinning in Relation to Event-Based Prospective Memory Performance Three Months after Moderate to Severe Traumatic Brain Injury in Children. 2011;35(3):318-332. doi:10.1080/87565641003696866.Patterns.

[21] Du A-T, Schuff N, Kramer JH, et al. Different regional patterns of cortical thinning in Alzheimer's disease and frontotemporal dementia. Brain. 2007;130(Pt 4):1159-1166. doi:10.1093/brain/awm016.

[22] Seo SW, Ahn J, Yoon U, et al. Cortical thinning in vascular mild cognitive impairment and vascular dementia of subcortical type. J Neuroimaging. 2010;20(1):37-45. doi:10.1111/j.1552-6569.2008.00293.x.

[23] Jovicich J, Czanner S, Greve D, et al. Reliability in multi-site structural MRI studies: Effects of gradient non-linearity correction on phantom and human data. Neuroimage. 2006;30(2):436-443. doi:10.1016/j.neuroimage.2005.09.046.

[24] Kothari PD, White NS, Farid N, et al. Longitudinal restriction spectrum imaging is resistant to pseudoresponse in patients with high-grade gliomas treated with bevacizumab. AJNR Am J Neuroradiol. 2013;34(9):1752-1757. doi:10.3174/ajnr.A3506.

[25] Thompson PM, Hayashi KM, Zubicaray G De, et al. Dynamics of Gray Matter Loss in Alzheimer' s Disease. J Neurosci. 2003;23(3):994-1005.

[26] RTOG. Phase III Double-Blind Placebo-Controlled Trial of Conventional Concurrent Chemoradiation and Adjuvant Temozolomide Plus Bevacizumab versus Conventional Concurrent Chemoradiation and Adjuvant Temozolomide in Patients with Newly Diagnosed Glioblastoma. Radiat Ther Oncol Gr RTOG 0825 Protoc Inf. 2014.

[27] Kwa SL, Lebesque J V., Theuws JCM, et al. Radiation pneumonitis as a function of mean lung dose: an analysis of pooled data of 540 patients. Int J Radiat Oncol Biol Phys. 1998;41(1):101-108. 
[28] Gondi V, Tomé WA, Mehta MP. Why avoid the hippocampus? A comprehensive review. Radiother Oncol. 2010;97(3):370-376. doi:10.1016/j.radonc.2010.09.013.

[29] Aoyama H, Kamada K, Shirato H, et al. Visualization of the corticospinal tract pathway using magnetic resonance axonography and magnetoencephalography for stereotactic irradiation planning of arteriovenous malformations. Radiother Oncol. 2003;68(1):27-32. doi:S016781400300032X [pii].

[30] RTOG. Randomized Phase II Trial of Hypofractionated Dose-Escalated Photon IMRT or Proton Beam Therapy Versus Conventional Photon Irradiation With Concomitant and Adjuvant Temozolomide in Patients With Newly Diagnosed Glioblastoma. Radiat Ther Oncol Gr NRG-BN001 Protoc Inf. 2014.

https://www.rtog.org/ClinicalTrials/ProtocolTable/StudyDetails.aspx?study= 1326. 
Figure 1. Probability of radiation-induced cortical atrophy for increasing grades of complication. Shaded bands represent $95 \%$ confidence intervals.

Figure 2. Example of cortical radiation sparing in a high grade glioma patient. Dose maps, color coded from 30 to $60 \mathrm{~Gy}$, are shown at various slice locations with (region-specific) and without (original) cortical sparing.

Figure 3. Histogram of the probability of atrophy for cortical voxels in a representative patient, using the original treatment plan, region-specific dose avoidance strategy, and non-specific dose avoidance. 
Table 1. Estimates, with $95 \% \mathrm{Cl}$, of $\gamma, D_{50}, D_{20}$, and $D_{10}$ for increasing grades of complication (Grade 1, 2, and 3).

\begin{tabular}{lccc}
\hline Parameter & grade 1 & grade 2 & grade 3 \\
$\gamma$ & 0.29 & 0.62 & 1.24 \\
& $(0.19-0.47)$ & $(0.53-0.96)$ & $(1.14-1.74)$ \\
$\mathrm{R}_{50}, \mathrm{~Gy}$ & 16.88 & 29.78 & 39.77 \\
& $(12.58-21.18)$ & $(27.08-33.04)$ & $(37.37-42.36)$ \\
$\mathrm{R}_{20}, \mathrm{~Gy}$ & & 13.60 & 28.65 \\
& & $(9.63-20.51)$ & $(27.38-32.36)$ \\
$\mathrm{R}_{10}, \mathrm{~Gy}$ & & 4.13 & 22.15 \\
& & $(0-13.52)$ & $(20.73-27.25)$
\end{tabular}


Table 2. Summary of the average change in dosimetric indices for regional and non-specific dose avoidance strategies. Cortical $V_{Y}$ decreased for all dose levels, irrespective of sparing strategy. Values are tabulated as mean [range].

Average change in dosimetric index for region-specific (RS) and non-specific (NS) approaches

\begin{tabular}{|c|c|c|c|c|c|c|c|c|c|c|}
\hline \multirow[t]{2}{*}{ Organ Type } & \multicolumn{3}{|c|}{ D2 } & \multicolumn{4}{|c|}{ D98 } & \multicolumn{3}{|c|}{$\mathrm{HI}$} \\
\hline & $\mathrm{RS}$ & & NS & & $\mathrm{RS}$ & NS & & $\mathrm{RS}$ & & NS \\
\hline \multirow[t]{3}{*}{ PTV } & $\begin{array}{r}0.8 \\
{[-0.2,1}\end{array}$ & & $\begin{array}{c}0.3 \\
{[-0.3,1.1]}\end{array}$ & & $\begin{array}{l}0.2 \\
2,0.8]\end{array}$ & $\begin{array}{r}-0.1 \\
{[-0.7,0}\end{array}$ & & $\begin{array}{c}1.9 \\
{[0.5,4.5]}\end{array}$ & & $\begin{array}{l}1.0 \\
6,2.9]\end{array}$ \\
\hline & \multicolumn{2}{|c|}{ V10 } & \multicolumn{2}{|c|}{ V20 } & \multicolumn{2}{|c|}{ V30 } & \multicolumn{2}{|c|}{ V40 } & \multicolumn{2}{|c|}{ V50 } \\
\hline & $\mathrm{RS}$ & NS & $\mathrm{RS}$ & NS & $\mathrm{RS}$ & NS & $\mathrm{RS}$ & NS & $\mathrm{RS}$ & NS \\
\hline Cortex & $\begin{array}{c}-2.3 \\
{[-11,0.2]}\end{array}$ & $\begin{array}{c}-3.6 \\
{[-17,0]}\end{array}$ & $\begin{array}{c}-3.6 \\
{[-5.3,-1.6]}\end{array}$ & $\begin{array}{c}-3.7 \\
{[-6.0,-1.6]}\end{array}$ & $\begin{array}{c}-4.6 \\
{[-9.5,-1.7]}\end{array}$ & $\begin{array}{c}-3.6 \\
{[-8.4,-0.6]}\end{array}$ & $\begin{array}{c}-3.1 \\
{[-5.8,-1.9]}\end{array}$ & $\begin{array}{c}-1.9 \\
{[-3.5,-0.5]}\end{array}$ & $\begin{array}{c}-1.6 \\
{[-2.8,-0.7]}\end{array}$ & $\begin{array}{c}-0.7 \\
{[-1.4,0]}\end{array}$ \\
\hline White Matter & $\begin{array}{c}-1.8 \\
{[-18,5.8]}\end{array}$ & $\begin{array}{c}-4.2 \\
{[-24,2.4]}\end{array}$ & $\begin{array}{c}-2.6 \\
{[-7.5,0.5]}\end{array}$ & $\begin{array}{c}-3.8 \\
{[-7,-1.3]}\end{array}$ & $\begin{array}{c}-3.1 \\
{[-6.3,0.2]}\end{array}$ & $\begin{array}{c}-4.1 \\
{[-7.6,-1.0]}\end{array}$ & $\begin{array}{c}-1.48 \\
{[2.3,-0.5]}\end{array}$ & $\begin{array}{c}-2.1 \\
{[-3.6,-0.5]}\end{array}$ & $\begin{array}{c}-0.8 \\
{[-1.5,-0.1}\end{array}$ & $\begin{array}{c}-0.8 \\
{[-1.4,-0.2]}\end{array}$ \\
\hline Hippocampus & $\begin{array}{c}0.1 \\
{[-7.1,8.5]}\end{array}$ & $\begin{array}{c}0.6 \\
{[-18,19]}\end{array}$ & $\begin{array}{c}-0.6 \\
{[-4.8,5.5]}\end{array}$ & $\begin{array}{c}-0.9 \\
{[-9.1,6.4]}\end{array}$ & $\begin{array}{c}-0.7 \\
{[-3.7,3.4]}\end{array}$ & $\begin{array}{c}-1.4 \\
{[-6.2,3.3]}\end{array}$ & $\begin{array}{c}-0.7 \\
{[-3.2,3.6]}\end{array}$ & $\begin{array}{c}-3.0 \\
{[-11,1.2]}\end{array}$ & $\begin{array}{c}0.3 \\
{[-3.2,3.9]}\end{array}$ & $\begin{array}{c}-0.9 \\
{[-5.7,2.1]}\end{array}$ \\
\hline
\end{tabular}





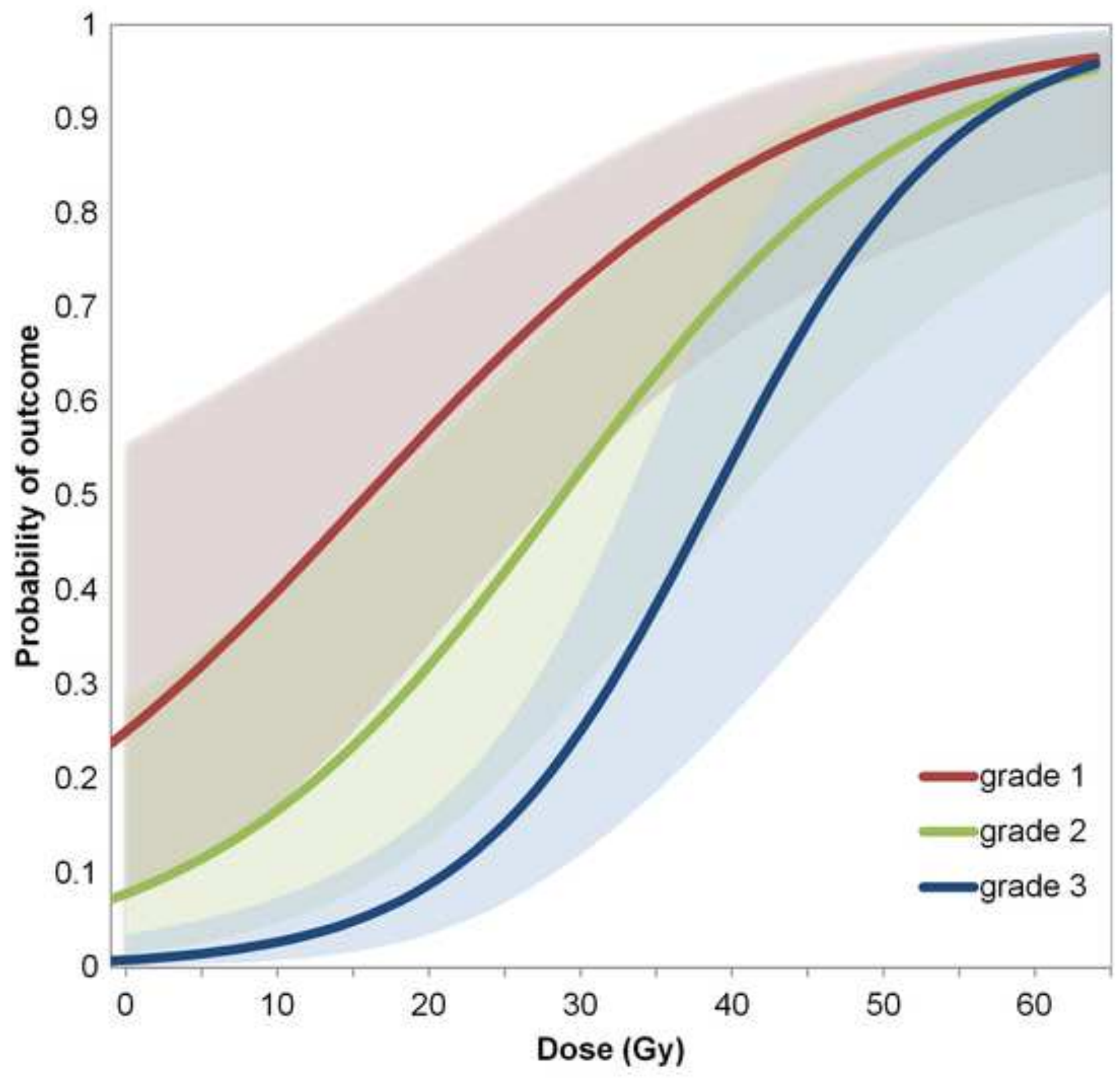


Slice Location
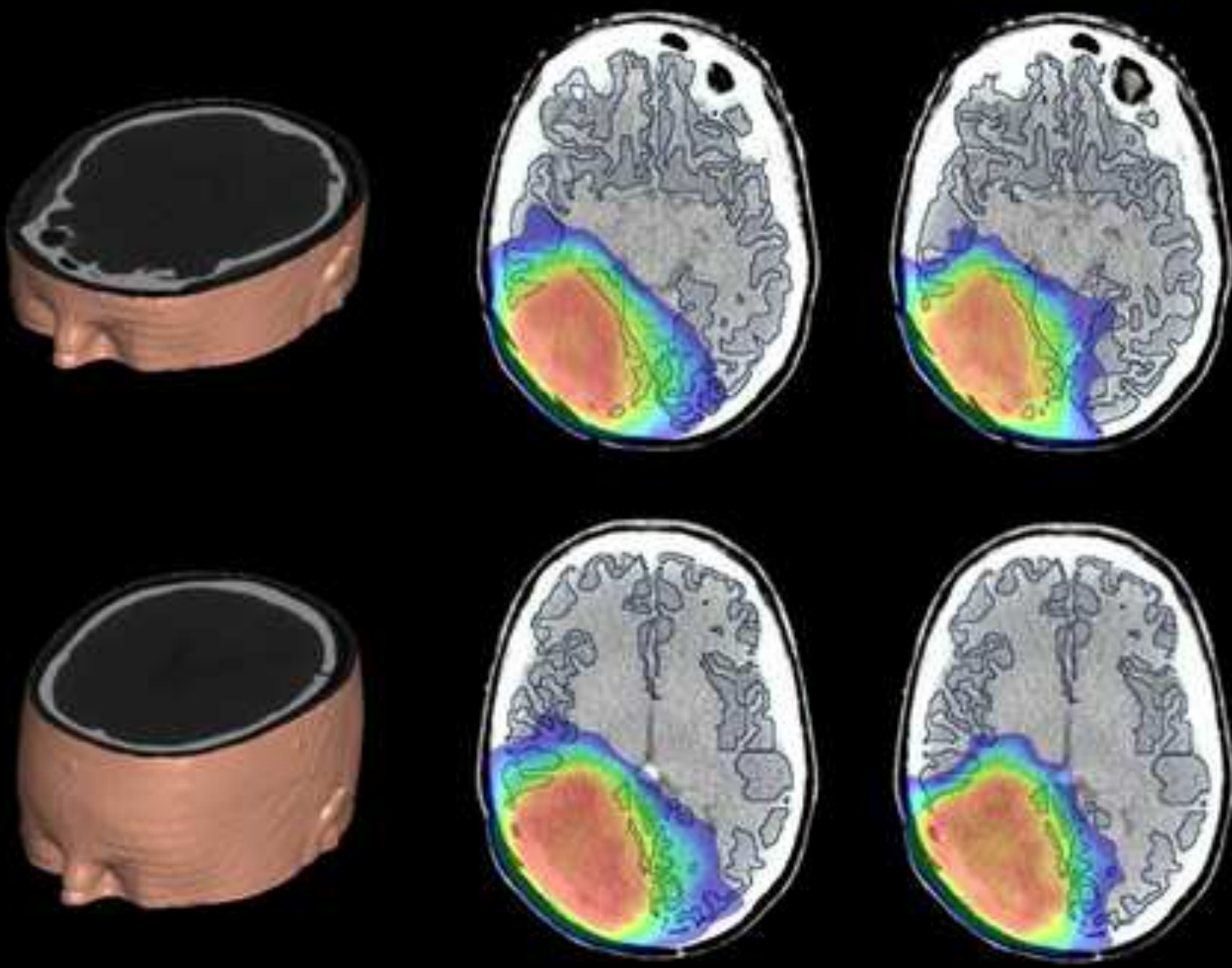
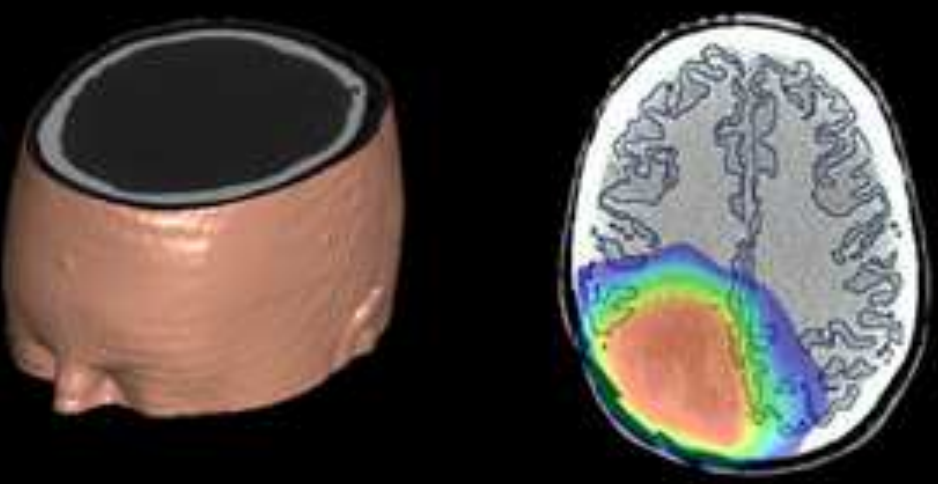

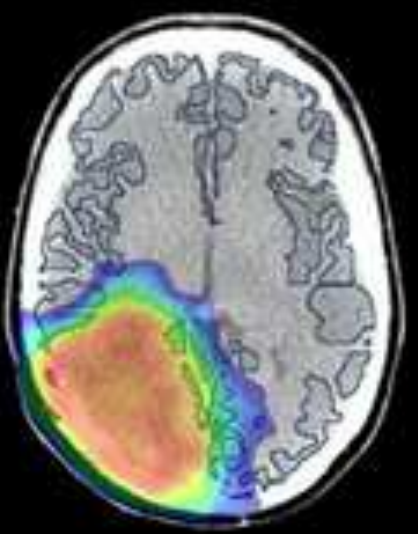

30 Gy

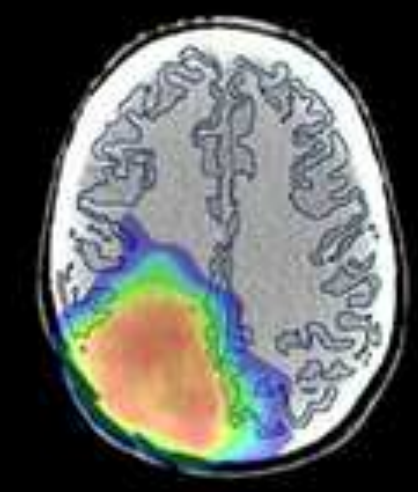

65 Gy

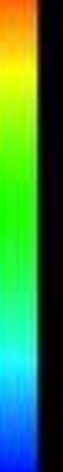




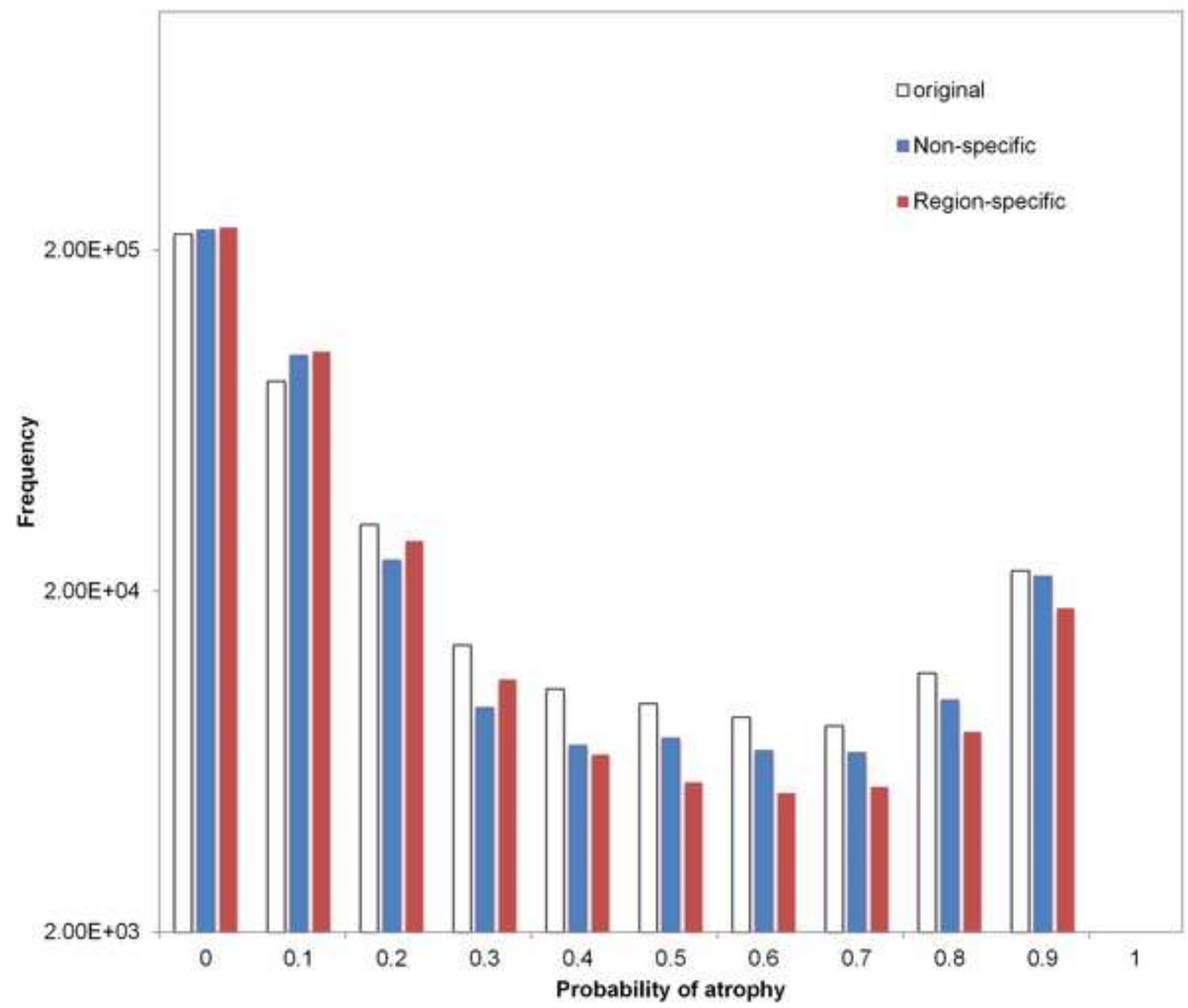

